\title{
Black hole entropy from the boundary conformal structure in 3D gravity with torsion
}

\author{
M. Blagojević ${ }^{1,2}$ and B. Cvetković ${ }^{1, *}$ \\ ${ }^{1}$ Institute of Physics, P.O.Box 57, 11001 Belgrade, Serbia \\ ${ }^{2}$ Department of Physics, Univ. of Ljubljana, Jadranska 19, 1000 Ljubljana, Slovenia
}

\begin{abstract}
Asymptotic symmetry of the Euclidean 3D gravity with torsion is described by two independent Virasoro algebras with different central charges. Elements of this boundary conformal structure are combined with Cardy's formula to calculate the black hole entropy.
\end{abstract}

\section{Introduction}

Thermodynamic properties of black holes, the gravitational objects with a highly complex dynamical structure, are expected to give us important clues to the quantum nature of gravity [1, 2]. Of particular importance for the development of these ideas have been the outstanding contributions achieved in the context of three-dimensional (3D) gravity 3 , 4, 15, 6, 7, 8, 9, 10, a simple model for exploring basic features of the gravitational dynamics. Traditionally, 3D gravity is formulated as general relativity with a cosmological constant $\left(\mathrm{GR}_{\Lambda}\right)$, with an underlying Riemannian structure of spacetime. In the early 1990s, Mielke and Baekler formulated a more general model - the model for 3D gravity with torsion based on Riemann-Cartan geometry [11. Such an approach might give us a new insight into the relationship between geometry and the dynamical structure of gravity.

Further development along these lines led to a number of interesting results [12, 13, 14, 15. 16, 17, 18, 19, 20. In particular, it was shown that: (a) the Mielke-Baekler model in Minkowskian spacetime possesses the black hole solution, and (b) for suitable boundary conditions, its asymptotic symmetry is described by two independent Virasoro algebras with different central charges [13, 16, 17, 19]. One is now tempted to use this Minkowskian asymptotic structure and calculate the black hole entropy in the manner of Strominger [10]. However, we prefer to base our study on the Euclidean formalism, where Cardy's formula, needed in the calculation, has a natural statistical interpretation [21, 22].

In the present paper, we study the Euclidean version of the Mielke-Baekler (MB) model and show that its asymptotic symmetry is locally isomorphic to the asymptotic symmetry of the Minkowskian theory. After this step, we are able to combine the resulting asymptotic

*E-mail: mb@phy.bg.ac.yu and cbranislav@phy.bg.ac.yu 
conformal structure with Cardy's formula for the asymptotic density of states of a boundary CFT [21, 22 and calculate the black hole entropy. The result is in complete agreement with the calculations based on the gravitational partition function 20]. This agreement is wellknown in $\mathrm{GR}_{\Lambda}$, but here, its validity is extended to the general MB model.

The layout of the paper is as follows. In Sect. II, we present basic aspects of the Euclidean MB model for 3D gravity with torsion, including the form of the black hole solution. In Sect. III, we discuss the asymptotic conditions and find the corresponding restrictions on the original gauge parameters. In Sect. IV, we find that the Poisson bracket algebra of the Euclidean theory is asymptotically conformal, and in Sect. V, we combine the boundary conformal structure with Cardy's formula to derive the black hole entropy. Sect. VI is devoted to concluding remarks, while appendices contain some technical details.

Our conventions are the same as in Ref. [20]: the Latin indices $(i, j, k, \ldots)$ refer to the local orthonormal frame, the Greek indices $(\mu, \nu, \rho, \ldots)$ refer to the coordinate frame, and both run over $0,1,2 ; \eta_{i j}=(+,+,+)$ are metric components in the local frame; totally antisymmetric tensor $\varepsilon^{i j k}$ and the related tensor density $\varepsilon^{\mu \nu \rho}$ are both normalized by $\varepsilon^{012}=+1$.

\section{Euclidean 3D gravity with torsion}

We begin with a brief account of the MB model in the Euclidean formalism, as a preparation for our treatment of the black hole entropy via Cardy's formula [20, 21].

Euclidean 3D gravity with torsion can be formulated as a gauge theory of the Euclidean group $I S O(3)$. In this approach, gauge potentials corresponding to local translations and local rotations are the triad field $b^{i}$ and the spin connection $\omega^{i}$ (1-forms), and the corresponding field strengths are the torsion and the curvature (2-forms): $T^{i}:=d b^{i}+\varepsilon^{i}{ }_{j k} \omega^{j} \wedge b^{k}$, $R^{i}:=d \omega^{i}+\frac{1}{2} \varepsilon^{i}{ }_{j k} \omega^{j} \wedge \omega^{k}$. In local coordinates $x^{\mu}$, the gauge potentials are represented as $b^{i}=b_{\mu}^{i} d x^{\mu}, \omega^{i}=\omega^{i}{ }_{\mu} d x^{\mu}$, and gauge transformations are parametrized by $\xi^{\mu}$ and $\theta^{i}$ :

$$
\begin{aligned}
\delta_{0} b^{i}{ }_{\mu} & =-\varepsilon^{i}{ }_{j k} b^{j}{ }_{\mu} \theta^{k}-\left(\partial_{\mu} \xi^{\rho}\right) b^{i}{ }_{\rho}-\xi^{\rho} \partial_{\rho} b^{i}{ }_{\mu}, \\
\delta_{0} \omega^{i}{ }_{\mu} & =-\nabla_{\mu} \theta^{i}-\left(\partial_{\mu} \xi^{\rho}\right) \omega^{i}{ }_{\rho}-\xi^{\rho} \partial_{\rho} \omega^{i}{ }_{\mu},
\end{aligned}
$$

where $\nabla_{\mu} \theta^{i}=\partial_{\mu} \theta^{i}+\varepsilon^{i}{ }_{j k} \omega^{j}{ }_{\mu} \theta^{k}$. The metric has the form $g=\eta_{i j} b^{i} \otimes b^{j}$, with $\eta_{i j}=\operatorname{diag}(1,1,1)$.

The geometric structure of $I S O(3)$ gauge theory corresponds to Riemann-Cartan geometry (for more details, see, for instance, Refs. [23, 24]).

The action integral. Mielke and Baekler proposed a topological model for 3D gravity in Riemann-Cartan spacetime [11, which is a natural generalization of $\mathrm{GR}_{\Lambda}$. The model is based on the action

$$
\bar{I}=a I_{1}+\Lambda I_{2}+\alpha_{3} I_{3}+\alpha_{4} I_{4}+I_{m},
$$

where $I_{m}$ is a matter contribution, and

$$
\begin{aligned}
& I_{1}:=2 \int b^{i} \wedge R_{i}, \\
& I_{2}:=-\frac{1}{3} \int \varepsilon_{i j k} b^{i} \wedge b^{j} \wedge b^{k}, \\
& I_{3}:=\int\left(\omega^{i} \wedge d \omega_{i}+\frac{1}{3} \varepsilon_{i j k} \omega^{i} \wedge \omega^{j} \wedge \omega^{k}\right), \\
& I_{4}:=\int b^{i} \wedge T_{i} .
\end{aligned}
$$


Here, the first two terms are of the same form as in $\mathrm{GR}_{\Lambda}, a=1 / 16 \pi G$ and $\Lambda$ is the cosmological constant, $I_{3}$ is the Chern-Simons action for the connection, and $I_{4}$ is a torsion counterpart of $I_{1}$.

The Euclidean MB action (2.2) is obtained from its Minkowskian counterpart [19] by the process of analytic continuation, and consequently, the parameters $\left(a, \Lambda, \alpha_{3}, \alpha_{4}\right)$ are expressed in terms of the corresponding Minkowskian quantities, as shown in Appendix A. The standard form of the action (2.2) is convenient for calculations, but at the end, we shall always use (A.2) to return to the Minkowskian parameters.

In the sector $\alpha_{3} \alpha_{4}-a^{2} \neq 0$, the vacuum field equations are non-degenerate:

$$
2 T^{i}=p \varepsilon^{i}{ }_{j k} b^{j} \wedge b^{k}, \quad 2 R^{i}=q \varepsilon^{i}{ }_{j k} b^{j} \wedge b^{k},
$$

where

$$
p:=\frac{\alpha_{3} \Lambda+\alpha_{4} a}{\alpha_{3} \alpha_{4}-a^{2}}, \quad q:=-\frac{\left(\alpha_{4}\right)^{2}+a \Lambda}{\alpha_{3} \alpha_{4}-a^{2}} .
$$

Introducing the Lavi-Civita connection $\tilde{\omega}^{i}$ by $d b^{i}+\varepsilon^{i}{ }_{m n} \tilde{\omega}^{m} b^{n}=0$, the field equations imply that Riemannian piece of the curvature $R^{i}(\tilde{\omega})$ has the form

$$
2 R^{i}(\tilde{\omega})=\Lambda_{\mathrm{eff}} \varepsilon^{i}{ }_{j k} b^{j} \wedge b^{k}, \quad \Lambda_{\mathrm{eff}}:=q-\frac{1}{4} p^{2},
$$

where $\Lambda_{\text {eff }}$ is the effective cosmological constant. Thus, our spacetime has maximally symmetric metric; it is known as the hyperbolic 3D space $H^{3}$, and has the isometry group $S O(3,1)$. In what follows, we will restrict our attention to the sector with positive $\Lambda_{\text {eff }}$, which represents the Euclidean continuation of anti-de Sitter space [20]. Accordingly, we use the notation $\Lambda_{\text {eff }}=: 1 / \ell^{2}$.

The black hole solution. For $\Lambda_{\text {eff }}>0$, equation (2.4) has a well known solution for the metric, the Euclidean BTZ black hole [6, 8]. In Schwarzschild-like coordinates $x^{\mu}=(t, r, \varphi)$, the metric has the form

$$
\begin{aligned}
& d s^{2}=N^{2} d t^{2}+N^{-2} d r^{2}+r^{2}\left(d \varphi+N_{\varphi} d t\right)^{2} \\
& N^{2}:=\left(-8 G m+\frac{r^{2}}{\ell^{2}}-\frac{16 G^{2} J^{2}}{r^{2}}\right), \quad N_{\varphi}:=-\frac{4 G J}{r^{2}} .
\end{aligned}
$$

The zeros of $N^{2}, r_{+}$and $r_{-}=-i \rho_{-}$, are related to the black hole parameters $m$ and $J$ by the relations $r_{+}^{2}-\rho_{-}^{2}=8 G m \ell^{2}, r_{+} \rho_{-}=4 G J \ell$, both $\varphi$ and $t$ are periodic,

$$
0 \leq \varphi<2 \pi, \quad 0 \leq t<\beta, \quad \beta=\frac{2 \pi \ell^{2} r_{+}}{r_{+}^{2}+\rho_{-}^{2}}
$$

and the black hole manifold is topologically a solid torus.

Starting with the BTZ metric (2.5), one can find the pair $\left(b^{i}, \omega^{i}\right)$ which solves the field equations (2.3), and represents the Euclidean black hole with torsion [20]:

$$
\begin{aligned}
b^{0} & =N d t, \quad b^{1}=N^{-1} d r, \quad b^{2}=r\left(d \varphi+N_{\varphi} d t\right), \\
\omega^{i} & =\tilde{\omega}^{i}+\frac{p}{2} b^{i},
\end{aligned}
$$


where the Levi-Civita connection $\tilde{\omega}^{i}$ reads:

$$
\tilde{\omega}^{0}=N d \varphi, \quad \tilde{\omega}^{1}=-N^{-1} N_{\varphi} d r, \quad \tilde{\omega}^{2}=-\frac{r}{\ell^{2}} d t+r N_{\varphi} d \varphi .
$$

Formally, the substitution $8 G m=-1, J=0$, "transforms" the black hole solution into the hyperbolic space $H^{3}$, the Euclidean analogue of $\mathrm{AdS}_{3}$. These solutions are locally isometric, but they have different topological properties.

\section{Asymptotic conditions}

Asymptotic conditions are an essential element of every field theory. In the canonical formalism, they are needed for the construction of well-defined symmetry generators and the related conserved charges.

Asymptotic configuration. Our choice of the asymptotic conditions in the MB model is essentially based on the arguments formulated twenty years ago by Brown and Henneaux in the context of $\mathrm{GR}_{\Lambda}$ [4]: they should be (a) sufficiently general to include the black hole configuration and (b) allow for the action of $\mathrm{SO}(3,1)$, the isometry group of $H^{3}$, but (c) sufficiently regular to yield well-defined canonical generators.

The asymptotic configuration of the triad field $b^{i}{ }_{\mu}$ that satisfies (a) and (b) is given by

$$
b_{\mu}^{i}=\left(\begin{array}{ccc}
\frac{r}{\ell}+\mathcal{O}_{1} & \mathcal{O}_{4} & \mathcal{O}_{1} \\
\mathcal{O}_{2} & \frac{\ell}{r}+\mathcal{O}_{3} & \mathcal{O}_{2} \\
\mathcal{O}_{1} & \mathcal{O}_{4} & r+\mathcal{O}_{1}
\end{array}\right)
$$

Here, for any $\mathcal{O}_{n}=c / r^{n}$, we assume that $c$ is not a constant, but a function of $t$ and $\varphi, c=c(t, \varphi)$, which is the simplest way to ensure the global $S O(3,1)$ invariance. This assumption is crucial for a highly non-trivial structure of the resulting asymptotic symmetry.

The asymptotic form of $\omega^{i}{ }_{\mu}$ is chosen in accordance with the field equations:

$$
\omega^{i}{ }_{\mu}=\left(\begin{array}{ccc}
\frac{p r}{2 \ell}+\mathcal{O}_{1} & \mathcal{O}_{4} & \frac{r}{\ell}+\mathcal{O}_{1} \\
\mathcal{O}_{2} & \frac{p \ell}{2 r}+\mathcal{O}_{3} & \mathcal{O}_{2} \\
-\frac{r}{\ell^{2}}+\mathcal{O}_{1} & \mathcal{O}_{4} & \frac{p r}{2}+\mathcal{O}_{1}
\end{array}\right) .
$$

A verification of the third condition (c) is left for the next section.

Asymptotic parameters. Having chosen the asymptotic conditions, we now wish to find the subset of gauge transformations (2.1) that respect these conditions. They are defined by restricting the original gauge parameters in accordance with (3.1), which yields:

$$
\begin{aligned}
& \xi^{0}=\ell\left[T-\frac{1}{2}\left(\frac{\partial^{2} T}{\partial t^{2}}\right) \frac{\ell^{4}}{r^{2}}\right]+\mathcal{O}_{4}, \\
& \xi^{2}=S-\frac{1}{2}\left(\frac{\partial^{2} S}{\partial \varphi^{2}}\right) \frac{\ell^{2}}{r^{2}}+\mathcal{O}_{4}, \\
& \xi^{1}=-\ell\left(\frac{\partial T}{\partial t}\right) r+\mathcal{O}_{1},
\end{aligned}
$$


and

$$
\begin{aligned}
\theta^{0} & =\frac{\ell^{2}}{r}\left(\frac{\partial^{2} T}{\partial t \partial \varphi}\right)+\mathcal{O}_{3}, \\
\theta^{2} & =-\frac{\ell^{3}}{r}\left(\frac{\partial^{2} T}{\partial t^{2}}\right)+\mathcal{O}_{3}, \\
\theta^{1} & =\left(\frac{\partial T}{\partial \varphi}\right)+\mathcal{O}_{2} .
\end{aligned}
$$

Here, $T$ and $S$ are functions that satisfy the conditions

$$
\ell \frac{\partial S}{\partial t}=-\frac{\partial T}{\partial \varphi}, \quad \frac{\partial S}{\partial \varphi}=\ell \frac{\partial T}{\partial t},
$$

which can be interpreted as the Cauchy-Riemann equations of complex analysis. Indeed, after introducing the complex variables

$$
w^{ \pm}:=\varphi \pm i \frac{t}{\ell}, \quad T^{ \pm}:=S \pm i T,
$$

one can rewrite these conditions as

$$
\partial_{-} T^{+}=0, \quad \partial_{+} T^{-}=0 .
$$

Consequently, general solutions for $T^{ \pm}$have the form $T^{+}=g\left(w^{+}\right), T^{-}=h\left(w^{-}\right)$, where $g, h$ are some analytic/anti-analytic functions.

The commutator algebra. The commutator of two gauge transformations (2.1) is closed: $\left[\delta_{0}^{\prime}, \delta_{0}^{\prime \prime}\right]=\delta_{0}^{\prime \prime \prime}$, where $\delta_{0}^{\prime}=\delta_{0}\left(\xi^{\prime}, \theta^{\prime}\right)$ and so on. The composition of parameters reads:

$$
\begin{aligned}
& \xi^{\prime \prime \prime} \mu=\xi^{\prime \rho} \partial_{\rho} \xi^{\prime \prime \mu}-\xi^{\prime \prime \rho} \partial_{\rho} \xi^{\prime \mu}, \\
& \theta^{\prime \prime \prime i}=\varepsilon^{i}{ }_{m n} \theta^{\prime m} \theta^{\prime \prime n}+\xi^{\prime} \cdot \partial \theta^{\prime \prime i}-\xi^{\prime \prime} \cdot \partial \theta^{i} .
\end{aligned}
$$

Applying the composition law to the restricted parameters (3.2) and keeping only the lowestorder terms, one finds the relations

$$
T^{\prime \prime \prime \pm}=T^{\prime \pm} \partial_{2} T^{\prime \prime \pm}-T^{\prime \prime \pm} \partial_{2} T^{\prime \pm}
$$

If we introduce the notation

$$
\begin{aligned}
& \delta_{n}^{-}:=\delta_{0}\left(T^{-}=e^{i n w^{-}}, T^{+}=0\right), \\
& \delta_{n}^{+}:=-\delta_{0}\left(T^{+}=e^{-i n w^{+}}, T^{-}=0\right),
\end{aligned}
$$

the commutator algebra takes the form

$$
\left[\delta_{n}^{\mp}, \delta_{m}^{\mp}\right]=-i(n-m) \delta_{n+m}^{\mp} .
$$

In general, the commutator algebra implies that the commutator of two $(T, S)$ transformations produces not only a $(T, S)$ transformation, but also an additional, pure gauge transformation (for which $T=S=0$ ). This result motivates us to introduce an improved definition of the asymptotic symmetry: it is the symmetry defined by the parameters (3.2), modulo the pure gauge transformations. Locally, this symmetry coincides with the conformal symmetry on the 2 -dimensional torus. The subalgebra generated by $n, m=0, \pm 1$, is so $(3,1)$, as expected. 


\section{Canonical realization of the asymptotic symmetry}

In this section, we use the canonical formalism to study the Poisson bracket algebra of the asymptotic symmetry.

\subsection{The improved canonical generator}

Following the standard Hamiltonian analysis, one can construct the gauge generator $G$, which produces the correct gauge transformations of all phase-space variables [25]. The transformation law of the fields, defined by $\delta_{0} \phi:=\{\phi, G\}$, is in complete agreement with the gauge transformations (2.1) on shell [20].

The gauge generator $G$ is constructed as a spatial integral of linear combinations of the first-class constraints, so that it vanishes on the constraint hypersurface: $G \approx 0$. Moreover, it acts on dynamical variables via the Poisson bracket operation, which is defined in terms of functional derivatives. On a manifold with boundary, $G$ does not have well-defined functional derivatives, but the problem can be cured by adding suitable surface terms 26. The improved canonical generator $\tilde{G}$ for the Euclidean MB model has the form:

$$
\tilde{G}=G+\Gamma, \quad \Gamma:=-\int_{0}^{2 \pi} d \varphi\left(\xi^{0} \mathcal{E}^{1}+\xi^{2} \mathcal{M}^{1}\right)
$$

where

$$
\begin{aligned}
& \mathcal{E}^{1}=2\left[\left(a+\frac{\alpha_{3} p}{2}\right) \omega^{0}{ }_{2}+\left(\alpha_{4}+\frac{a p}{2}\right) b^{0}{ }_{2}-\frac{a}{\ell} b^{2}{ }_{2}-\frac{\alpha_{3}}{\ell} \omega^{2}{ }_{2}\right] b_{0}^{0}, \\
& \mathcal{M}^{1}=2\left[\left(a+\frac{\alpha_{3} p}{2}\right) \omega^{2}{ }_{2}+\left(\alpha_{4}+\frac{a p}{2}\right) b_{2}^{2}+\frac{a}{\ell} b^{0}{ }_{2}+\frac{\alpha_{3}}{\ell} \omega^{0}{ }_{2}\right] b_{2}{ }_{2} .
\end{aligned}
$$

The adopted asymptotic conditions guarantee differentiability and finiteness of $\tilde{G}$. Moreover, $\tilde{G}$ is also conserved.

Conserved charges. The value of the improved generator $\tilde{G}$ defines the gravitational charge. Since $\tilde{G} \approx \Gamma$, the charge is completely determined by the boundary term $\Gamma$. Note that $\Gamma=0$ for the pure gauge transformations.

For $\xi^{2}=0, \tilde{G}$ reduces to the time translation generator, while for $\xi^{0}=0$ we obtain the spatial rotation generator. The corresponding surface terms, calculated for $\xi^{0}=1$ and $\xi^{2}=1$, respectively, have the meaning of energy and angular momentum:

$$
E=-\int_{0}^{2 \pi} d \varphi \mathcal{E}^{1}, \quad M=-\int_{0}^{2 \pi} d \varphi \mathcal{M}^{1} .
$$

Energy and angular momentum are conserved gravitational charges. Their values for the black hole configuration (3.2) are

$$
E=i\left[m+\frac{\alpha_{3}}{a}\left(\frac{p m}{2}-\frac{J}{\ell^{2}}\right)\right], \quad M=i\left[J+\frac{\alpha_{3}}{a}\left(\frac{p J}{2}+m\right)\right] .
$$

The conserved charges in the MB model are linear combinations of the $\mathrm{GR}_{\Lambda}$ charges $m, J$. Returning to the Minkowskian parameters with the help of Appendix A, we find

$$
E=i\left[m+\frac{\alpha_{3}}{a}\left(\frac{p m}{2}-\frac{J}{\ell^{2}}\right)\right]^{\mathrm{M}}, \quad M=-\left[J+\frac{\alpha_{3}}{a}\left(\frac{p J}{2}-m\right)\right]^{\mathrm{M}},
$$

or equivalently, $E=i E^{\mathrm{M}}$ and $M=-M^{\mathrm{M}}$. 


\subsection{Canonical algebra}

In the canonical formalism, the asymptotic symmetry is determined by the Poisson bracket algebra of the improved generators $\tilde{G}$. In the notation $G^{\prime}:=G\left[T^{\prime}, S^{\prime}\right], G^{\prime \prime}:=G\left[T^{\prime \prime}, S^{\prime \prime}\right]$, and so on, the Poisson bracket algebra is found to have the form

$$
\left\{\tilde{G}^{\prime \prime}, \tilde{G}^{\prime}\right\}=\tilde{G}^{\prime \prime \prime}+C^{\prime \prime \prime},
$$

where the parameters $\left(T^{\prime \prime \prime}, S^{\prime \prime \prime}\right)$ are determined by the composition rules (3.4), and $C^{\prime \prime \prime}$ is the classical central term:

$$
\begin{aligned}
C^{\prime \prime \prime}= & -\left(2 a+\alpha_{3} p\right) \ell \int_{0}^{2 \pi} d \varphi\left(\partial_{2} S^{\prime \prime} \partial_{2}^{2} T^{\prime}-\partial_{2} S^{\prime} \partial_{2}^{2} T^{\prime \prime}\right) \\
& +2 \alpha_{3} \int_{0}^{2 \pi} d \varphi\left(\partial_{2} T^{\prime \prime} \partial_{2}^{2} T^{\prime}-\partial_{2} S^{\prime \prime} \partial_{2}^{2} S^{\prime}\right) .
\end{aligned}
$$

The Poisson bracket algebra (4.4) can be brought into a more familiar form by introducing the generators

$$
\begin{aligned}
& L_{n}^{-}:=-\tilde{G}\left(T^{-}=e^{i n w^{-}}, T^{+}=0\right), \\
& L_{n}^{+}:=\tilde{G}\left(T^{+}=e^{-i n w^{+}}, T^{-}=0\right),
\end{aligned}
$$

which produce the transformations with parameters $T^{-}=e^{i n w^{-}}$and $T^{+}=e^{-i n w^{+}}$, respectively. These generators obey two independent Virasoro algebras with central charges:

$$
\begin{aligned}
\left\{L_{m}^{\mp}, L_{n}^{\mp}\right\} & =-i(m-n) L_{n+m}^{\mp}-\frac{i c^{\mp}}{12} m^{3} \delta_{m+n, 0}, \\
i c^{\mp} & :=24 \pi\left[a \ell+\alpha_{3}\left(\frac{p \ell}{2} \mp i\right)\right]
\end{aligned}
$$

and $\left\{L_{n}^{-}, L_{m}^{+}\right\}=0$. The algebra (4.6a) is a central extension of the commutator algebra (3.5). Returning to the Minkowskian parameters by using (A.2), we find

$$
c^{\mp}=24 \pi\left[a \ell+\alpha_{3}\left(\frac{p \ell}{2} \mp 1\right)\right]^{\mathrm{M}},
$$

which are exactly the values found earlier in the Minkowskian formalism [19]. The boundary CFT is characterized by different classical central charges, corresponding to holomorphic and antiholomorphic sectors. The asymptotic Poisson bracket algebra for the Euclidean MB theory is isomorphic to the corresponding Minkowskian algebra.

\section{Black hole entropy via Cardy's formula}

Using the definitions (4.5), we can express the values of $L_{0}^{\mp}$ in terms of the conserved charges:

$$
L_{0}^{\mp} \approx \mp \frac{1}{2}(M \pm i \ell E)=\frac{1}{2}\left(\ell E^{\mathrm{M}} \pm M^{\mathrm{M}}\right)=: h^{\mp} .
$$


Now, we can apply Cardy's formula for the asymptotic density of states of a boundary CFT and calculate the black hole entropy. The formula is based entirely on the asymptotic symmetry structure expressed by the Virasoro algebra (4.6), and has the form:

$$
S=2 \pi \sqrt{\frac{h^{-} c^{-}}{6}}+2 \pi \sqrt{\frac{h^{+} c^{+}}{6}},
$$

where $h^{\mp}$ are given in terms of the Minkowskian parameters as

$$
h^{\mp}=(\ell m \pm J)^{\mathrm{M}} \frac{c^{\mp}}{48 \pi a \ell}=\frac{\left(r_{+} \pm r_{-}\right)^{2}}{8 G \ell} \frac{c^{\mp}}{48 \pi a \ell} .
$$

Thus, the entropy of the black hole with torsion takes the form (in units $\hbar=1$ ):

$$
\begin{aligned}
S & =\frac{\pi}{6 \ell}\left[r_{+}\left(c^{-}+c^{+}\right)+r_{-}\left(c^{-}-c^{+}\right)\right] \\
& =\frac{2 \pi r_{+}}{4 G}+4 \pi^{2} \alpha_{3}\left(p r_{+}-\frac{2 r_{-}}{\ell}\right) .
\end{aligned}
$$

This result, obtained from the boundary conformal structure, coincides with the gravitational entropy, based on the calculation of the grand canonical partition function [20].

In $\mathrm{GR}_{\Lambda}$, where $\alpha_{3}=0$, we have $S=2 \pi r_{+} / 4 G$, as expected. For $p=0$, our expression for $S$ reduces to Solodukhin's result [27], obtained in Riemannian geometry, but with a Chern-Simons term in the action.

Note that the second term in $S$, which represents the modification of the $\mathrm{GR}_{\Lambda}$ result, depends on both the outer and inner horizons, $r_{+}$and $r_{-}$. Since the Euclidean black hole manifold does not contain the inner horizon, its appearance in $S$ can be understood as a consequence of the analytic structure of the black hole solution: the Euclidean black hole "remembers" that its Minkowskian counterpart has the inner horizon. On the other hand, the presence of the Chern-Simons coupling constant $\alpha_{3}$ and the strength of torsion $p$ shows the influence of the geometric structure of spacetime on the gravitational dynamics.

Cardy's formula (5.2) holds under the following assumptions [22]:

(a) the boundary CFT is unitary: $h^{-} \geq 0, h^{+} \geq 0$ (this implies $c^{-}, c^{+} \geq 0$ );

(b) the values $h^{-}, h^{+}$are sufficiently large: $h^{-} \gg c^{-} / 24, h^{+} \gg c^{+} / 24$;

(c) the lowest possible values of $h^{-}, h^{+}$are zero: $h_{0}^{-}=0, h_{0}^{+}=0$.

With $c_{0}=24 \pi a \ell=3 \ell / 2 G$, the conditions (b) can be rewritten in the form $\ell m \pm J \gg c_{0} / 12$, which is the same as in $\mathrm{GR}_{\Lambda}$. Consequently, in the semiclassical regime $m$ has to be large in Planck units:

$$
m \gg 1 / \hbar G \text {. }
$$

Clearly, the fact that $S$ and $c^{\mp}$ are not allowed to take negative values imposes certain bounds on $\alpha_{3}, p$ and $r_{ \pm}$.

Cardy's formula (5.2) is obtained from the zero-loop (classical) approximation of the CFT partition function. The one-loop correction is obtained in a simmilar manner [28]; when applied to the MB model, it gives the one-loop correction to $S$ (Appendix B). 


\section{Concluding remarks}

In this paper, we found that the asymptotic symmetry of the Euclidean 3D gravity with torsion is the conformal symmetry, described by two independent Virasoro algebras with different central charges and locally isomorphic to the corresponding Minkowskian structure. The black hole entropy is then calculated using Cardy's formula for the asymptotic density of states of a boundary CFT. The result is in perfect agreement with the gravitational entropy, obtained via the gravitational partition function [20. This agreement, well-known for Riemannian $\mathrm{GR}_{\Lambda}$, is now generalized to the MB model of 3D gravity with torsion.

\section{Acknowledgements}

This work was supported by the Serbian and Slovenian Science Foundations.

\section{A Euclidean continuation}

In the process of analytic continuation, different terms of the Minkowskian MB action $I_{M}$ transform into their Euclidean counterparts according to the rule (Appendix A in [20]):

$$
\begin{aligned}
& b^{i} R_{i} \mapsto i b^{i} R_{i}, \quad b^{0} b^{1} b^{2} \mapsto-i b^{0} b^{1} b^{2}, \\
& \mathcal{L}_{\mathrm{CS}}(\omega) \rightarrow \mathcal{L}_{\mathrm{CS}}(\omega), \quad b^{i} T_{i} \rightarrow-b^{i} T_{i} .
\end{aligned}
$$

Consequently, the Euclidean action (2.2) is obtained from $I_{M}$ through the analytic continuation, $I_{M} \mapsto \bar{I}$, if its parameters $\left(a, \Lambda, \alpha_{3}, \alpha_{4}\right)$ are related to the Minkowskian parameters $\left(a^{\mathrm{M}}, \Lambda^{\mathrm{M}}, \alpha_{3}^{\mathrm{M}}, \alpha_{4}^{\mathrm{M}}\right)$ as follows:

$$
\begin{array}{ll}
a=i a^{\mathrm{M}}, & \Lambda=-i \Lambda^{\mathrm{M}}, \\
\alpha_{3}=\alpha_{3}^{\mathrm{M}}, & \alpha_{4}=-\alpha_{4}^{\mathrm{M}} .
\end{array}
$$

We also have $J^{\mathrm{M}}=-i J$. Note that A.2 differs from the result defined by the analytic continuation $I_{M} \mapsto i I_{E}$, discussed in Ref. [20], by the presence of an additional factor $i$.

\section{B The one-loop correction}

The one-loop correction to the black hole entropy can be found using the results of Ref. [28], according to which the corrected Cardy formula for the density of states has the form

$$
\rho\left(h^{-}, h^{+}\right)=\rho\left(h^{-}\right) \rho\left(h^{+}\right), \quad \rho\left(h^{\mp}\right) \approx\left[\frac{C^{\mp}}{96\left(h^{\mp}\right)^{3}}\right]^{1 / 4} \exp \left(2 \pi \sqrt{\frac{h^{\mp} C^{\mp}}{6}}\right),
$$

where $C^{\mp}$ is the full central charge, $C^{\mp}=c^{\mp}+$ quantum corrections. In the approximation $C^{\mp} \approx c^{\mp}$, taking the logarithm of $\rho\left(h^{-}, h^{+}\right)$leads to

$$
S=\frac{1}{\hbar} S^{(0)}-\frac{3}{2} \ln \left(\frac{2 \pi r_{+}}{\hbar G} \ell \kappa\right)+\text { const }+\mathcal{O}(\hbar),
$$

where $S^{(0)}$ is the classical value (15.3) , and $\kappa=\left(r_{+}^{2}-r_{-}^{2}\right) / \ell^{2} r_{+}$is is the surface gravity. The first-order correction in (B.1) has the same form as in $\mathrm{GR}_{\Lambda}$. 


\section{References}

[1] J. D. Bekenstein, Black holes and entropy, Phys. Rev. D 5, 2333 (1973);

S. W. Hawking, Black hole explosions, Nature 228, 30 (1974); Particle creation by black holes, Commun. Math. Phys. 43, 199 (1975).

[2] See, for instance, S. F. Ross, Black hole thermodynamics, hep-th/0502195.

[3] For a review of the subject and an extensive list of references, see: S. Carlip, Lectures on (2+1)-Dimensional Gravity, J. Korean Phys. Soc. 28, 447 (1995) [gr-qc/9503024]; Quantum Gravity in 2+1 Dimensions (Cambridge University Press, Cambridge, 1998); Conformal Field Theory, (2+1)-dimensional Gravity, and the BTZ Black Hole, Class. Quantum Grav. 22, R85-R124 (2005).

[4] J. D. Brown and M. Henneaux, Central Charges in the Canonical Realization of Asymptotic Symmetries: An Example from Three Dimensional Gravity, Comm. Math. Phys. 104, 207 (1986);

see also M. Henneaux, Energy-momentum, angular momentum, and superscharge in 2+1 dimensions, Phys. Rev. D 29, 2766 (1984).

[5] A. Achucarro and P. Townsend, A Chern-Simons Action For Three-Dimensional AntiDe Sitter Supergravity Theories, Phys. Lett. B 180, 89 (1986);

E. Witten, 2+1 dimensional gravity as an exactly soluble system, Nucl. Phys. B 311, $46(1988 / 89)$.

[6] M. Bañados, C. Teitelboim and J. Zanelli, The Black Hole in Three-Dimensional Spacetime, Phys. Rev. Lett. 16, 1849 (1993);

M. Bañados, M. Henneaux, C. Teitelboim and J. Zanelli, Geometry of $2+1$ Black Hole, Phys. Rev. D 48, 1506 (1993).

[7] O. Coussaert, M. Henneaux and P. van Driel, The asymptotic dynamics of threedimensional Einstein gravity with negative cosmological constant, Class. Quantum Grav. 12, 2961 (1995).

[8] M. Bañados, T. Brotz and M. Ortiz, Boundary dynamics and the statistical mechanics of the $2+1$ dimensional black hole, Nucl. Phys. B 545, 340 (1999);

M. Bañados, Three-dimensional quantum geometry and black holes, hep-th/9901148.

[9] S. Carlip and C. Teitelboim, Aspects of Black Hole Quantum Mechanics and Thermodynamics in 2+1 Dimensions, Phys. Rev. D 51, 622 (1995);

S. Carlip, The Statistical Mechanics of the Three-Dimensional Euclidean Black Hole, Phys. Rev. D 55, 878 (1997).

[10] A. Strominger, Black hole entropy from near-horizon microstates, JHEP 02, 009 (1998).

[11] E. W. Mielke, P. Baekler, Topological gauge model of gravity with torsion, Phys. Lett. A 156, 399 (1991);

P. Baekler, E. W. Mielke, F. W. Hehl, Dynamical symmetries in topological 3D gravity with torsion, Nuovo Cim. B 107, 91 (1992). 
[12] T. Kawai, Exotic black hole solutions in teleparallel theory of $(2+1)$-dimensional gravity, Prog. Theor. Phys. 94 (1995) 1169;

A. A. Sousa, J. W. Maluf, Black Holes in 2+1 Teleparallel Theories of Gravity, Prog. Theor. Phys. 104 (2000) 531.

[13] A. García, F. W. Hehl, C. Heinecke and A. Macías, Exact vacuum solutions of (1+2)dimensional Poincaré gauge theory: BTZ solution with torsion, Phys. Rev. D 67, 124016 (2003).

[14] Y. Obukhov, New solutions in 3D gravity, Phys. Rev. D 68, 124015 (2003).

[15] M. Blagojević M and M. Vasilić, Asymptotic symmetries in 3D gravity with torsion, Phys. Rev. D 67, 084032 (2003); Asymptotic dynamics in 3D gravity with torsion, Phys. Rev. D 68, 124007 (2003).

[16] M. Blagojević M and M. Vasilić, 3D gravity with torsion as a Chern-Simons gauge theory, Phys. Rev. D 68, 104023 (2003).

[17] E. W. Mielke and A. A. R. Maggiolo, Rotating black hole solution in a generalized topological 3D gravity with torsion, Phys. Rev. D 68, 104026 (2003).

[18] S. Cacciatori, M. Caldarelli, A. Giacomini, D. Klemm and D. Mansi, Chern-Simons formulation of three-dimensional gravity with torsion and nonmetricity, J. Geom. Phys. 56, 2523 (2006) [hep-th/0507200].

[19] M. Blagojević and B. Cvetković, Canonical structure of 3D gravity with torsion, in: Progress in General Relativity and Quantum Cosmology, vol. 2, ed. Ch. Benton (Nova Science Publishers, New York, 2006), p. 85 [gr-qc/0412134].

[20] M. Blagojević and B. Cvetković, Black hole entropy in 3D gravity with torsion, Class. Quantum Grav. 23, 4781 (2006).

[21] J. A. Cardy, Operator Content of Two-Dimensional Conformally Invariant Theories, Nucl. Phys. B 270, 186 (1986).

[22] S. Carlip, What We Don't Know about BTZ Black Hole Entropy, Class. Quantum Grav. 15, 3609 (1998).

[23] F. W. Hehl, Four lectures on Poincaré gauge theory, Proceedings of the 6th Course of the School of Cosmology and Gravitation, on Spin, Torsion, Rotation and Supergravity, held in Ericce, Italy, 1979, eds. P. G. Bergmann, V. de Sabbata (Plenum, New York, 1980), p. 5;

E. W. Mielke, Geometrodynamics of Gauge Fields - On the geometry of Yang-Mills and gravitational gauge theories (Akademie-Verlag, Berlin, 1987).

[24] M. Blagojević, Gravitation and gauge symmetries (Bristol, Institute of Physics Publishing, 2002);

Three lectures on Poincaré gauge theory, SFIN A 1, 147 (2003) [gr-qc/0302040)]. 
[25] L. Castellani, Symmetries of constrained Hamiltonian systems, Ann. Phys. (N.Y.) 143, 357 (1982).

[26] T. Regge and C. Teitelboim, Role of surface integrals in the Hamiltonian formulation of general relativity, Ann. Phys. (N.Y.) 88, 286 (1974).

[27] S. N. Solodukhin, Holography with Gravitational Chern-Simons Term, Phys. Rev. D 74, 02405 (2006).

[28] S. Carlip, Logarithmic corrections to black hole entropy from Cardy formula, Class. Quantum Grav. 17, 4175 (2000). 